\title{
Real Linear Substitutions with Lquimodular Multipliers, and their expression in terms of their Invariants.
}

\author{
By Dr. D. G. TAYLOR.
}

(Received 4th November 1915. Read 10th December 1915).

\section{Section I.-Introduction.}

§. Let

$$
x_{\alpha}^{\prime}=\sum_{\beta} l_{\alpha \beta} x_{\beta},(\alpha, \beta=1,2, \ldots n),
$$

denote a linear substitution of non-vanishing determinant; and let the roots $k_{\epsilon}$ of its characteristic equation

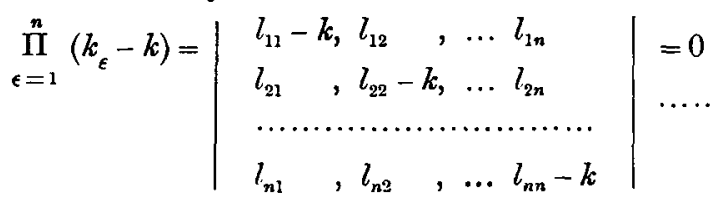

be for the present assumed distinct. Then with each root $k_{\epsilon}$ is associated an invariant point or pole $P_{\epsilon}$, and a linear invariant, or invariant $(n-2)$-plane $\xi_{\epsilon}$. If the $n$ points $P_{\epsilon}$ do not lie on an $(n-2)$-plane, the determinant of their coordinates,

$$
D=\left|\begin{array}{cccc}
x_{11}, & x_{21}, \ldots & x_{n 1} \\
x_{12}, & x_{22}, \ldots & x_{n 2} \\
\ldots \ldots \ldots \ldots \ldots \ldots \\
x_{1 n}, & x_{2 n}, \ldots . & x_{n n}
\end{array}\right|
$$

will be non-vanishing, where $x_{a, \epsilon}(\alpha=1,2, \ldots n)$ are the coordinates of $P_{\varepsilon}$; and the equation of the linear invariant associated with $k_{\epsilon}$ is

$$
\xi_{\varepsilon}=\sum_{a} X_{a \epsilon} x_{\alpha}=0
$$

$X_{\alpha \epsilon}$ denoting as usual the cofactor of $x_{\alpha \epsilon}$ in $D$. Since the linear invariants $\xi_{\epsilon}$, and also the coordinates of the poles $P_{\epsilon}$, are, on application of the substitution, reproduced unchanged, except that 
they are multiplied each by the associated root $k_{\epsilon}$ of the characteristic equation, these roots will be called the multipliers of the substitution.

With the assumptions stated, we at once obtain the formula

$$
D l_{a \beta}=\sum_{\epsilon} k_{\epsilon} \dot{x}_{\alpha \epsilon} X_{\beta \epsilon^{\prime}}(\alpha, \beta=1,2, \ldots n), \ldots \ldots \ldots \ldots
$$

which determine the coefficients of the substitution when its poles and multipliers are given. If we have two substitutions with the same poles, but with different multipliers $k_{\epsilon}, k_{\epsilon}^{\prime}(\epsilon=1,2, \ldots n)$, then if

$$
\lambda_{\alpha \beta}=c l_{\alpha \beta}+c^{\prime} l_{\alpha \beta}^{\prime}, \kappa_{\epsilon}=c k_{\epsilon}+c^{\prime} k_{\epsilon^{\prime}}^{\prime}
$$

$c, c^{\prime}$ being any constants, the substitution whose coefficients are $\left(\lambda_{a \beta}\right)$ will have the same poles as the given ones, and the quantities $\kappa_{\epsilon}$ for multipliers. Further, the effect of the substitution $\left(l_{\alpha \beta}\right)$ followed by $\left(l_{\alpha \beta}^{\prime}\right)$ will be to change the coordinates $x_{\alpha \epsilon}$ of the pole $P_{\epsilon}$ into $k_{\epsilon}^{\prime} k_{\epsilon} x_{\alpha \epsilon}$; that is, the product of the two substitutions is another substitution with the same poles, whose multiplers $K_{\epsilon}$ are respectively the products of the corresponding multipliers of the given ones, or

$$
K_{\epsilon}=k_{\epsilon}^{\prime} k_{\epsilon}, \quad(\epsilon=1,2, \ldots n) \text {. }
$$

Thus copolar substitutions are permutable; and also the result of repeating a given substitution $r$ times is a substitution with the same poles, and with the quantities $k_{\epsilon}^{r}$ for multipliers. If $l_{a \beta}^{(r)}$ denote a coefficient in the final substitution, then

$$
D l_{a \beta}^{(r)}=\sum_{\epsilon} k_{\epsilon}^{r} x_{\alpha \epsilon} X_{\beta \epsilon}
$$

and a complete index law can be established, negative and fractional indices included, for a substitution possessing $n$ inde. pendent poles.

In building up a substitution from its poles and multipliers, it is easy to trace the effect of an equality between two of the latter. If $k_{2}=k_{1}$, every point on the line $P_{1} P_{2}$ is a pole, and any two of them may be taken in place of the given two $P_{1}, P_{2}$. We can still find $n$ independent poles, though they are not unique; and the specification of the substitution in terms of poles and multipliers is not impaired. A similar argument holds when three or more multipliers are equal; and the linear invariants might be used instead of the poles in developing the argument. 
On the other hand when, in a substitution of which the coefficients are given, two of the multipliers are found to be equal, two cases may arise. The equations for the coordinates of the corresponding pole may yield a line of poles, or only a single pole. For example, the substitution

$$
\left.\begin{array}{l}
x_{1}^{\prime}=k x_{1} \\
x_{2}^{\prime}=l_{21} x_{1}+k x_{2} \\
x_{3}^{\prime}=l_{31} x_{1}+l_{33} x_{2}+k x_{3}^{\prime}
\end{array}\right\},
$$

of which the three multipliers are equal, possesses only one pole. In such a case the poles and multipliers are not sufficient to specify the substitution; but this case does not arise in the sequel.

\section{Section II.-Cyclant Substitutions.}

$\$ 2$. In any system of $n$ homogeneous point-coordinates there are $n$ special sets of values which may be said to define the unit-points of the system. They are

where

$$
P_{\epsilon}\left(x_{1 \epsilon}, x_{2 \epsilon}, \ldots x_{n \varepsilon}\right),(\epsilon=1,2, \ldots n) \text {, }
$$

$$
\rho^{\epsilon} x_{1 \epsilon}=\rho^{2 \epsilon} x_{2 \epsilon}=\ldots=\rho^{(n-1) \epsilon} x_{n-3, \epsilon}=x_{n, \epsilon}
$$

$\rho$ being any primitive $n$th root of unity. We may assume $\rho=\exp (2 \pi i / n)$, since any other admissible value would yield the same points, only in a different order. The $(n-2)$-plane passing through all of these points except $P_{\epsilon}$ is

$$
\xi_{\epsilon}=\sum_{g=1}^{n} \rho^{8 \epsilon} x_{s}=0
$$

Consider now the "cyclant" substitution *

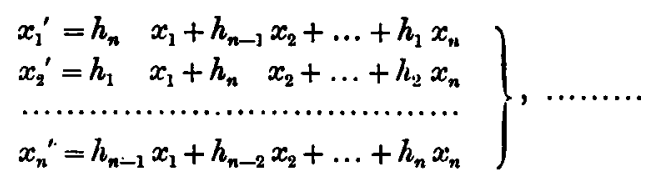

where each row on the right contains the same $n$ coefficients in the same cyclic order, with the same coefficient $h_{n}$ always in the leading

* Hilton, Linear Substitutions, p. 12, Ex. 7. 
diagonal. The value of its determinant is equal to the product of $n$ factors linear in the $h_{8}$, which may be written *

$$
k_{\epsilon}=\sum_{s=1}^{n} \rho^{s \epsilon} h_{s},(\epsilon=1,2, \ldots n),
$$

$\rho$ being as before a primitive $n$th root of unity. The quantities $k_{\epsilon}$ are also the roots of its characteristic equation ; and it can easily be verified that the pole and linear invariant associated with $k_{\epsilon}$ as multiplier are the unit-point $P_{\epsilon}$ and the $(n-2)$-plane $\xi_{\epsilon}$ just defined. Since equations (10) can be solved uniquely for the $h_{g}$ in terms of the $k_{\epsilon}$, it follows that every linear substitution possessing the unitpoints as poles is of the form (9). The actual form of the results is

$$
n h_{s}=\sum_{\epsilon=1}^{n} \rho^{-8 \epsilon} k_{\epsilon}, \quad(s=1,2, \ldots n) .
$$

Since the unit points are all different, every substitution of type (9) can be completely specified in terms of its poles and multipliers; and it is not necessary that the latter be all different.

In general the coefficients, multipliers, and variables of (9) are alike complex quantities. When the multipliers are all different, only one of the liuear invariants, namely $\xi_{n}$, has real coefficients when $n$ is odd ; and only two, namely $\xi_{z n}, \xi_{n}$, when $n$ is even. But when equalities occur among the multipliers, additional linear invariants with real coefficients may arise, as will appear later.

Since substitutions with zero determinant are excluded, none of the multipliers can vanish. They cannot all be equal, else the substitution becomes a "similarity-substitution." $\dagger$ By reducing the coefficients in a constant ratio, any one of the multipliers, say $k_{n}$, can be made equal to unity.

When two of the multipliers, say $k_{\epsilon^{\prime}} k_{\epsilon^{\prime}}$, are equal, any linear function of the corresponding invariants, say $\lambda \xi_{e}+\mu \xi_{e^{\prime}}$, where $\lambda, \mu$ are constants, is also invariant with the same multiplier. In particular let $k_{\epsilon}=k_{n-\epsilon}$; then it is clear from (8) that there are two additional linear invariants with real coefficients, namely,

$$
\xi_{\varepsilon}+\xi_{n-\epsilon}, i\left(\xi_{\varepsilon}-\xi_{n-\epsilon}\right) \text {. }
$$

* Scott, Determinants, p. 81.

† Hilton, ibid., p. 26 
Let the coefficients $k_{a}$ be all real. Then by (10) $k_{n}$ is real, and also $k_{\frac{1}{2} n}$ when $n$ is even; while $k_{\epsilon}, k_{n-\epsilon}$ are conjugate imaginaries, or, as a special case, real and equal. The imaginary linear invariants can then be combined into quadratic invariants with real coefficients, of the form

$$
\xi_{\epsilon} \xi_{n-\epsilon} \text {, multiplier }\left|k_{\epsilon}\right|^{2}, \quad(\epsilon=1,2, \ldots p),
$$

where $p=\frac{1}{2}(n-1)$ or $\frac{1}{2}(n-2)$ according as $n$ is odd or even. These real quadratic invariants are characteristic of the case in which the coefficients of the substitution are real.

If in addition to the coefficients being real, the multipliers have all the same modulus, then the quadratic invariants have all the same multiplier. 'This is the case of equimodular multipliers discussed below.

If we impose the further restriction that the amplitudes of the multipliers shall be all of the form $2 s \pi / r(s, r$ integers), then the mutual ratios of the multipliers will be $r^{\text {th }}$ roots of unity, and the substitution of finite order $r$.

$\$ 3$. The treatment of the relations between the coefficients in the case of equimodular multipliers will be facilitated by the following notation. Placing the coefficients $h_{1}, h_{2}, \ldots h_{n}$ in order round a circle, multiply each by that coefficient which is $s$ places in advance of it, and denote the sum of such products by $H_{n}$, thus :

$$
H_{s}=h_{1} h_{s+1}+h_{2} h_{s+2}+\ldots+h_{n} h_{s} ;
$$

$H_{0}$ or $H_{n}$ may be used indifferently for the quantity $\sum_{s} h_{*}^{2}$. Let $t$ stand for $\frac{1}{2}(n-1)$ or $\frac{1}{2} n$ according as $n$ is odd or even. Then there will be $(t+1)$ distinct functions $H_{s}$, defined by $s=0,1, \ldots t$. Each will consist of a sum of $n$ products; except $H_{\frac{1}{n} n}$ in the case of $n$ even, which will contain only half the number; thus for $n=4$,

$$
H_{2}=h_{1} h_{3}+h_{2} h_{4} \text {. }
$$

This notation, explained for the coefficients $h$, may be used also for the variables $x$ and multipliers $k$.

We shall prove that when the coefficients are real and the multipliers equimodular, $H_{0}$ is equal to the square of the common modulus, and the other expressions $H_{\mathrm{s}}$ vanish.

Let the common modulus be $\kappa$; then equating $\kappa^{2}$ to $\left|k_{\epsilon}\right|^{2}$ as obtained from $(10)$, we obtain the $(t+1)$ equations

$\left(H_{0}-\kappa^{2}\right)+2 H_{1} \cos \frac{2 \epsilon \pi}{n}+\ldots+2 H_{t} \cos \frac{2 t \epsilon \pi}{n}=0,(\epsilon=0,1, \ldots t) \ldots$ 
Now it is easily seen that the determinant of the trigonometrical coefficients of these equations does not vanish; hence

$$
\left.\begin{array}{l}
H_{1}=H_{2}=\ldots=H_{t}=0 \\
H_{0}=\kappa^{2}=k_{n}^{2}=(\Sigma h)^{2}
\end{array}\right\}
$$

\$4. The Quadratic Invariants. Assuming only, to begin with, that the coetficients $h_{t}$ are real, we have, as alruady seen, the following quadratic invariants with real coefticients :

$$
\begin{aligned}
& \xi_{\epsilon} \xi_{n-\epsilon}, \text { multiplier }\left|k_{\epsilon}\right|^{2},(\epsilon=1,2, \ldots t), \\
& \xi_{n}{ }^{2}, \quad, \quad k_{n}{ }^{2} .
\end{aligned}
$$

Noting the similarity in form between (8) and (10) we have on the analogy of (13)

$$
\xi_{\epsilon} \xi_{n-\epsilon}=X_{0}+2 X_{1} \cos \frac{2 \epsilon \pi}{n}+\ldots+2 X_{t} \cos \frac{2 t \epsilon \pi}{n},(\epsilon=0,1, \ldots t) \ldots
$$

where the case $\epsilon=0$ corresponds to $\hat{\zeta}_{n}{ }^{2}$ just above. These expressions constitute the $(t+1)$ quadratic invariants with real coefficients which exist independently of any equalities among the multipliers $k_{\epsilon}$ or their moduli.

Now let the multipliers be equimodular. Then these invariants all possess the same modulus $H_{0}$; hence any linear function of them is also an invariant with the same modulus. It follows that, when the coefficients are real and the multipliers equimodular, the $(t+1)$ quadratic forms $X_{0}, X_{1}, \ldots X_{t}$ are invariants with common multiplier $H_{0}$.

If we consider the set of multipliers

$$
k_{\epsilon}=\rho^{p \epsilon},(\epsilon=1,2, \ldots n),
$$

where $p$ is an integer, we find from (11) that the corresponding coefticients $h_{\text {a }}$ all vanish except $h_{p}$, which has the value unity. The substitution thus reduces to the cyclic permutation

$$
x_{r}^{\prime}=x_{n-p+r},(r=1,2, \ldots n) ;
$$

and conversely every cyclic permutation of the letters $x$ corresponds to a set of multipliers of form (16).

If $x_{n} y_{r}(r=1,2, \ldots n)$ are two sets of variables subjected to substitutions of the form (9), but with different coefficients, and if

$$
V_{r}=\sum_{r=1}^{n} x_{s} y_{r \rightarrow s}
$$


then it is found that the substitution which transforms the quantities $V_{r}$ is equivalent to the product of those which transform the $x_{r}, y_{r}$.

If, further, the $x_{r}, y_{r}$ are subjected to the same substitution, and that substitution satisfy the conditions (14) for equimodular multipliers, then the quantities

$$
U_{r}=\sum_{s=1}^{n} x_{s} y_{r+t},(r=1,2, \ldots n)
$$

are invariants with the same multiplier as the $X_{r}, Y_{r}$.

$\$ 5$. In the case of three variables, on the hypothesis that the coefficients are real and the multipliers equimodular, we may without loss put

we then have by (14)

$$
k_{1}=e^{i \theta}, \quad k_{2}=e^{-i \theta}, \quad k_{3}=1 ;
$$

$$
h_{1}{ }^{2}+h_{2}{ }^{2}+h_{3}{ }^{2}=1, \quad h_{2} h_{3}+h_{3} h_{1}+h_{1} h_{2}=0 ;
$$

the coefficients can then be written in the form

$$
h_{s}=\frac{1}{3}\{2 \cos (\theta-2 s \pi / 3)+1\},(s=1,2,3) ;
$$

the substitution will be of finite order $r$, provided $\theta$ is of the form $2 p \pi / r$, where $p, r$ are integral, and the quadratic invariants are

$$
X_{0}=x_{1}^{2}+x_{2}^{2}+x_{3}^{2}, \quad X_{1}=x_{2} x_{3}+x_{3} x_{1}+x_{1} x_{2},
$$

each with multiplier unity.

Let $\left(x_{1}, x_{2}, x_{3}\right),\left(y_{1}, y_{2}, y_{3}\right)$ be the direction-cosines of two lines with respect to trirectangular axes; and let them be transformed by the above substitution. The following quantities will remain unchanged :

$$
\sum_{r} x_{r}^{2}, \sum_{r} y_{r}^{2}, \sum_{r} x_{r} y_{r}, \sum_{r} x_{r}, \sum_{r} y_{r},(r=1,2,3) .
$$

Thus the transformed quantities are also the direction-cosines of two lines, containing the same angle as the two original lines, and each making the same angle as its original with the line of symmetry $\left(\frac{1}{\sqrt{ } 3}, \frac{1}{\sqrt{ } 3}, \frac{1}{\sqrt{ } 3}\right)$. The substitution therefore represents a rotation through a definite angle about the line of symmetry.

It is easily shown that the amount of the rotation is $+\theta$, with the usual sign convention, where $\theta$ is the amplitude of the multiplier $k_{1}$. The rotation changes the $z$-axis into the line $\left(h_{1}, h_{2}, h_{3}\right)$, and similarly for the other axes. 
Developments of this application will appear later.

In the case of four variables, we have easily

$$
\left.\begin{array}{ll}
k_{1}+k_{3}=-2\left(h_{3}-h_{4}\right), & k_{1}-k_{3}=2 i\left(h_{1}-h_{3}\right) \\
k_{2}+k_{1}=2\left(h_{2}+h_{4}\right), & k_{3}-k_{4}=-2\left(h_{1}+h_{3}\right)
\end{array}\right\} ;
$$

and the conditions for equimodular multipliers are

$$
\begin{aligned}
& H_{1} \equiv h_{1} h_{2}+h_{2} h_{3}+h_{3} h_{4}+h_{4} h_{1} \equiv\left(h_{1}+h_{3}\right)\left(h_{2}+h_{4}\right)=0, \\
& H_{2} \equiv h_{1} h_{3}+h_{2} h_{4}=0 .
\end{aligned}
$$

We can therefore distinguish two cases :

(A) $k_{1}=e^{i \theta}, k_{3}=e^{-i \theta},-k_{2}=k_{4}=1$, with $h_{2}+h_{4}=0$; we then have

$$
\left.\begin{array}{l}
h_{1}=\frac{1}{2}(1+\sin \theta) \\
h_{2}=-\frac{1}{2} \cos \theta \\
h_{3}=\frac{1}{2}(1-\sin \theta) \\
h_{4}=\frac{1}{2} \cos \theta
\end{array}\right\}
$$

(B) $k_{1}=e^{i \theta}, k_{3}=e^{-i \theta}, k_{2}=k_{4}=1$, with $h_{1}+h_{3}=0$ ；

and then

$$
\left.\begin{array}{l}
h_{1}=\frac{1}{2} \sin \theta \\
h_{2}=\frac{1}{2}(1-\cos \theta) \\
h_{3}=-\frac{1}{2} \sin \theta \\
h_{4}=\frac{1}{2}(1+\cos \theta)
\end{array}\right\}
$$

It will be found that in the first case there are the quadratic invariants

$$
\begin{array}{cc}
X_{0}, X_{1}, X_{2}, & \text { multiplier 1, } \\
\left(x_{1}+x_{3}\right)^{2}-\left(x_{4}+x_{4}\right)^{2}, & , \quad-1 ;
\end{array}
$$

while in the second, in virtue of the equality of $k_{3}, k_{4}$, the quadratic invariants are

$$
X_{0}, X_{1}, X_{2},\left(x_{1}+x_{3}\right)^{2},\left(x_{2}+x_{4}\right)^{2} \text {, multiplier } 1 \text {. }
$$

On analogy with three dimensions, let any two sets of quantities $x_{r}, y_{r}(r=1,2,3,4)$ satisfying the condition

$$
\sum x^{2}=\sum y^{2}=1
$$

be called the direction cosines of two lines in four-space. Since

$$
\sum x^{2} \cdot \sum y^{2}-\left(\sum_{r} x_{r} y_{r}\right)^{2}=\sum_{r, s}\left(x_{r} y_{s}-x_{s} y_{r}\right)^{2},
$$

the summation on the right extending to six terms, therefore $\sum_{r} x_{r} y_{r}$ is numerically less than unity, and may be called the cosine of the angle between the lines. The lines may be called parallel when this quantity is unity, and at right angles when it vanishes. 
We have just seen that, corresponding to any value of the parameter $\theta$, there are two distinct real equimodular linear substitutions of form (9) in four variables. The operation in four dimensions which corresponds to a rotation $\theta$ about the line of symmetry in three dimensions is ambiguous.

Now the interpretation of what we may call mode (B) above is simple. Rotation in four dimensions takes place not about a line but about a plane, and the plane is determined by two lines lying in it. It is clear that in mode (B) the quantities

$$
\sum_{r} x_{r}, x_{1}+x_{3}, x_{2}+x_{4}
$$

are all invariant, with multiplier +1 ; hence the substitution indicates a rotation about the plane determined by any two of the three lines through the origin whose direction-cosines are

$$
\left(\frac{1}{2}, \frac{1}{2}, \frac{1}{2}, \frac{1}{2}\right),\left(\frac{1}{\sqrt{ } 2}, 0, \frac{1}{\sqrt{ } 2}, 0\right),\left(0, \frac{1}{\sqrt{ } 2}, 0, \frac{1}{\sqrt{ } 2}\right) ;
$$

these three lines evidently lying in one plane.

In mode ( $d$ ), on the other hand, there is but one linear invariant with multiplier +1 , namely $\xi_{\downarrow} \equiv \sum_{r} x_{r}$. The invariant

$$
\xi_{2} \equiv x_{1}-x_{2}+x_{3}-x_{4}
$$

has multiplier -1 . If the line $\left(x_{1}, x_{2}, x_{3}, x_{4}\right)$ makes angles $\phi_{1}, \phi_{2}$ with the lines $\left(\frac{1}{2}, \frac{1}{2}, \frac{1}{2}, \frac{1}{2}\right),\left(\frac{1}{2},-\frac{1}{2}, \frac{1}{2},-\frac{1}{2}\right)$ respectively, then the line obtained from it by a substitution in mode (A) will make with the same two lines the respective angles $\phi_{1}, \pi-\phi_{2}$. We may for the moment call this a quasi-rotation about the plane determined by these lines. Though not itself a rotation, two repetitions of it are equivalent to two repetitions of mode (B), i.e. to a rotation of amount $2 \theta$ about the plane indicated above.

When $n=5$, the conditions for real coefficients and equimodular multipliers show that we can put

$$
k_{1}=e^{i \theta_{1}}, k_{2}=e^{i \theta_{2}}, k_{3}=e^{-i \theta_{2}}, k_{4}=e^{-i \theta_{1}}, k_{5}=1 ;
$$

that the real quadratic invariants are $X_{0}, X_{1}, X_{2}$; and the coefficients can be written down in terms of $\theta_{1}, \theta_{2}$.

Thus, as to the analogue in five dimensions of a rotation about the line of symmetry in three dimensions, two parameters $\theta_{1}, \theta_{2}$ are 
necessary for its specification; but for given values of these parameters the operation is determined without ambiguity.

When $n=6$, then by (14)

whence

$$
H_{1}=H_{2}=H_{3}=0 \text {, }
$$

$$
\left(h_{1}+h_{3}+h_{5}\right)\left(h_{2}+h_{4}+h_{6}\right) \equiv H_{1}+H_{3}=0,
$$

yielding, as with $n=4$, an ambiguous case.

(A) With $h_{2}+h_{4}+h_{6}=0$, we have $k_{3}=-k_{6}$, and the real quadratic invariants

$$
\begin{array}{ccc}
X_{0}, X_{1}, X_{2}, X_{3}, & \text { multiplier } & +1, \\
\left(x_{1}+x_{3}+x_{5}\right)^{2}-\left(x_{2}+x_{4}+x_{6}\right)^{2}, & , & -1 .
\end{array}
$$

(B) With $h_{1}+h_{3}+h_{5}=0$, we have $k_{3}=k_{6}$, and the real quadratic invariants, all with multiplier +1 ,

$$
X_{0}, X_{1}, X_{2}, X_{3},\left(x_{1}+x_{3}+x_{5}\right)^{2},\left(x_{2}+x_{4}+x_{6}\right)^{2} \text {. }
$$

We can now generalize as follows:

In the case of $n$ real variables, with equimodular multipliers, the coefficients satisfy the conditions

$$
H_{0}=1, H_{1}=H_{2}=\ldots=H_{t}=0,
$$

and the real quadratic functions

$$
X_{0}, X_{1}, \ldots X_{t}
$$

are invariant, multiplier +1 ; where $t=\frac{1}{2}(n-1)$ or $\frac{1}{2} n$, according as $n$ is odd or even. When $n$ is odd, the number of angular parameters $\theta$ required to specify the substitution is $\frac{1}{2}(n-1)$, and, these being given, the substitution is unique.

When $n$ is even, the number required is $\frac{1}{2}(n-2)$; but, these being even, the substitution is ambiguous. In the second or (B) mode in such cases the functions

$$
\left(\sum x_{2 r+1}\right)^{2}, \quad\left(\Sigma x_{2 r}\right)^{2}
$$

are also invariants with multiplier +1 ; in the first or (A) mode, the difference of these functions is an invariant with multiplier -1 .

Section III.-Construction of Real Linear Substitutions of Equimodular Type from their Real Linear and Quadratic Invariants.

$\$ 6$. It is proposed to extend to more general cases some of the results obtained in last section for cyclant substitutions. 
The coefficients of the characteristic equation being real functions of the coefficients of the substitution, the imaginary multipliers of a real substitution must occur in conjugate pairs. It follows that the imaginary linear invariants can also be arranged in pairs, such that the product of the two members of a pair is a real quadratic invariant.

Thus the invariants of a real substitution can always be given as one or two ${ }^{*}$ real linear, and $\frac{1}{2}(n-1)$ or $\frac{1}{2}(n-2)$ real quadratic invariants, according as $n$ is odd or even As a rule each quadratic invariant must be the product of two conjugate imaginary linear invariants; but this will not of necessity be so when equalities subsist among the multipliers or their moduli. In particular, when all the multipliers are equimodular, the quadratic functions whose linear factors constitute the imaginary linear invariants, need not be the given quadratic invariants, but linear combinations of these, and of the square of the real linear invariant (or of the squares and products of the two or more real linear invariants, when such exist).

§7. $n=3$.

In the case of a real substitution in three variables with equimodular multipliers, the most general assumption for the linear invariants is

$$
\begin{aligned}
& \xi_{1}=\left(p_{1}+i q_{1}\right) x_{1}+\left(p_{2}+i q_{2}\right) x_{2}+\left(p_{3}+i q_{3}\right) x_{3}, \text { mult. } e^{i \theta}
\end{aligned}
$$

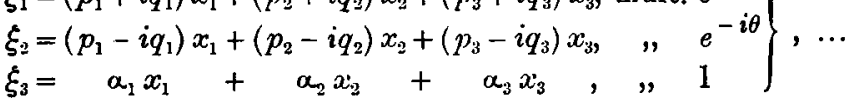

where the $p, q, \alpha$ are real.

The coordinates of the poles, being proportional to the minors of the determinant of the coefficients of (19), are as follows, where

$$
\left(p_{r} q_{s}\right) \equiv p_{r} q_{s}-p_{s} q_{r}, \text { etc : }
$$

$$
\left.\begin{array}{l}
P_{1}: \frac{x_{11}}{\left(p_{2} \alpha_{3}\right)-i\left(q_{2} \alpha_{3}\right)}=\frac{x_{21}}{\left(p_{3} \alpha_{3}\right)-i\left(q_{3} \alpha_{1}\right)}=\frac{x_{31}}{\left(p_{1} \alpha_{2}\right)-i\left(q_{1} \alpha_{2}\right)} \\
P_{2}: \frac{x_{12}}{-\left(p_{2} \alpha_{3}\right)-i\left(q_{2} \alpha_{3}\right)}=\frac{x_{22}}{-\left(p_{3} \alpha_{1}\right)-i\left(q_{3} \alpha_{1}\right)}=\frac{x_{32}}{-\left(p_{1} \alpha_{2}\right)-i\left(q_{1} \alpha_{2}\right)} \\
P_{3}: \frac{x_{13}}{-2 i\left(p_{2} q_{3}\right)}=\frac{x_{23}}{-2 i\left(p_{3} q_{1}\right)}=\frac{x_{33}}{-2 i\left(p_{1} q_{2}\right)}
\end{array}\right\}
$$

* For a case with no real linear invariants, but $\frac{1}{2} n$ real quadratic invariants, see below, $\S 10$. 
If we write

$$
D_{0} \equiv\left|\begin{array}{ccc}
p_{1}, & p_{2}, & p_{3} \\
q_{1}, & q_{2}, & q_{3} \\
\alpha_{1}, & \alpha_{2}, & \alpha_{3}
\end{array}\right|,
$$

then the determinant of the coefficients in (19) is equal to $-2 i D_{0}$, and that of the coordinates in (20) is

$$
-4 D_{0}^{2}=D \text {, say. }
$$

Now applying formula (5),

$$
D l_{r 4}=\left(x_{r 1} X_{s 1}+x_{r 2} X_{s 2}\right) \cos \theta+\left(x_{r 1} X_{s 1}-x_{r 2} X_{s 2}\right) i \sin \theta+x_{r 3} X_{\star 3} ;
$$

so that for $r \neq s$,

$$
D l_{r s}=x_{r 3} X_{s 3}(1-\cos \theta)+i\left(x_{r 1} X_{s 1}-x_{r 2} X_{s 2}\right) \sin \theta \text {, }
$$

while

$$
D l_{r r}=D \cos \theta+x_{r 3} X_{r 3}(1-\cos \theta)+i\left(x_{r 1} X_{r 1}-x_{r 2} X_{r 2}\right) \sin \theta \text {. }
$$

For the $x$ we may take the actual denominators in (20); and then for each $X$ we must take $-2 i D_{0}$ times the corresponding coefficient in (19).

Putting for short

$$
p_{r} p_{s}+q_{r} q_{s}=\delta_{r s}, \quad p_{r} q_{s}-p_{s} q_{r}=\epsilon_{r s},
$$

we have for the coefficients of the substitution the following :

$$
\left.\begin{array}{ll}
D_{0}\left(l_{11}-\cos \theta\right) & =\epsilon_{23} \alpha_{1}(1-\cos \theta)+\left(\delta_{13} \alpha_{2}-\delta_{22} \alpha_{3}\right) \sin \theta \\
D_{0} l_{12} & =\epsilon_{23} \alpha_{2}(1-\cos \theta)+\left(\delta_{23} \alpha_{2}-\delta_{22} \alpha_{3}\right) \sin \theta \\
D_{0} l_{13} & =\epsilon_{23} \alpha_{3}(1-\cos \theta)+\left(\delta_{33} \alpha_{2}-\delta_{32} \alpha_{3}\right) \sin \theta
\end{array}\right\},
$$

and the six others derived from these by cyclic interchange of the suffixes $1,2,3$.

As an example, let us suppose that in addition to the linear invariant $\Sigma \alpha x$, we are given the quadratic $\Sigma x^{2}$, (see above, $\S 6$ ) each with multiplier +1 . Then since $\Sigma x^{2}$ must, be a function of the linear invariants, there must exist a relation of the form

$$
(\Sigma a x)^{2}+\lambda \Sigma x^{2}=\mu\left\{(\Sigma p x)^{2}+(\Sigma q x)^{2}\right\} .
$$

The condition for the breaking-up of the left-hand member into factors yields

$$
\lambda=-\left(\alpha_{1}^{2}+\alpha_{2}^{2}+\alpha_{3}^{2}\right),
$$

and we then have, on equating coefficients,

$$
\left.\begin{array}{ll}
\alpha_{2}{ }^{2}+\alpha_{3}{ }^{2}=-\mu\left(p_{1}{ }^{2}+q_{1}{ }^{2}\right), & \alpha_{2} \alpha_{3}=\mu\left(p_{2} r_{3}+q_{2} q_{3}\right) \\
\alpha_{3}{ }^{2}+\alpha_{1}{ }^{2}=-\mu\left(p_{2}{ }^{2}+q_{3}{ }^{2}\right), & \alpha_{3} \alpha_{1}=\mu\left(p_{3} p_{1}+q_{3} q_{1}\right) \\
\alpha_{1}{ }^{2}+\alpha_{2}{ }^{2}=-\mu\left(p_{3}{ }^{2}+q_{3}{ }^{2}\right), & \alpha_{1} \alpha_{2}=\mu\left(p_{1} p_{2}+q_{1} q_{2}\right)
\end{array}\right\}
$$


We may without loss assume

then

$$
p_{3}=-\left(\alpha_{1}^{2}+\alpha_{2}^{2}\right), q_{3}=0, \alpha_{1}^{2}+\alpha_{2}^{2}+\alpha_{3}^{2}=1 ;
$$

$$
\mu=p_{3}^{-1}, p_{1}=\alpha_{3} \alpha_{1}, p_{2}=\alpha_{3} \alpha_{3}, q_{1}=\mp \alpha_{3}, q_{2}= \pm \alpha_{1} \text {. }
$$

For definiteness we may take the upper signs, since it is only a question of the order of the invariants $\xi_{1}, \xi_{2}$. Thus

$$
\left.\begin{array}{l}
\xi_{1}=\left(\alpha_{3} \alpha_{1}-i \alpha_{2}\right) x_{1}+\left(\alpha_{2} \alpha_{3}+i \alpha_{1}\right) x_{2}-\left(\alpha_{1}^{2}+\alpha_{2}^{2}\right) x_{3} \\
\xi_{2}=\left(\alpha_{3} \alpha_{1}+i \alpha_{2}\right) x_{1}+\left(\alpha_{2} \alpha_{3}-i \alpha_{1}\right) x_{2}-\left(\alpha_{1}^{2}+\alpha_{2}^{2}\right) x_{3}
\end{array}\right\},
$$

and the poles are

$$
\begin{aligned}
& P_{1}\left\{\alpha_{2}-i \alpha_{3} \alpha_{1},-\alpha_{1}-i \alpha_{2} \alpha_{3}, i\left(\alpha_{1}^{2}+\alpha_{2}^{2}\right)\right\} \\
& \left.\begin{array}{ccc}
P_{2}\left\{-\alpha_{2}-i \alpha_{3} \alpha_{1},\right. & \left.\alpha_{1}-i \alpha_{2} \alpha_{3}, i\left(\alpha_{1}^{2}+\alpha_{2}^{2}\right)\right\} \\
P_{3}\left(\alpha_{1},\right. & \alpha_{2}, & \alpha_{3}
\end{array}\right) \text {, }
\end{aligned}
$$

where the common factor $-2 i\left(\alpha_{1}{ }^{2}+\alpha_{2}{ }^{2}\right)$ has been discarded from the coordinates of $P_{3}$. The coefficients of the substitution can now be written down :

$$
\left.\begin{array}{ll}
l_{11}=\cos \theta+ & \alpha_{1}^{2}(1-\cos \theta) \\
l_{12}= & \alpha_{1} \alpha_{2}(1-\cos \theta)-\alpha_{3} \sin \theta \\
l_{13}= & \alpha_{1} \alpha_{3}(1-\cos \theta)+\alpha_{2} \sin \theta
\end{array}\right\},
$$

with the six others obtained by cyclic interchange of suffixes.

As was to be expected, these are the coefficients of the substitution undergone by the direction-cosines of a line which is rotated through the angle $\theta$ about an axis whose direction-cosines are $\left(a_{1}, \alpha_{2}, a_{3}\right)$. They agree with the formulae given in Whittaker, Analytical Dynamics, $\S 7$. They reduce to (17) above on putting $\alpha_{1}=\alpha_{2}=\alpha_{2}=1 / \sqrt{ } 3$. The general rotation-substitution in three dimensions bas thus been deduced purely from its multipliers and the invariants $\Sigma \alpha x, \Sigma x^{2}$; and its imaginary linear invariants and its poles are given in terms of the cosines of the axis by (23), (24).

Let us assume more generally that the function

$$
S \equiv a x_{1}^{2}+b x_{i}^{2}+c x_{3}^{2}+2 f x_{2} x_{3}+2 g x_{3} x_{1}+2 h x_{1} x_{2},
$$

in which the coefficients are real, is an invariant with multiplier unity, in addition to the real linear invariant $\xi_{3} \equiv \Sigma \alpha x$. As before, a relation must exist of the form

$$
\xi_{3}^{2}+\lambda S=\mu \xi_{1} \xi_{2}
$$


The value of $\lambda$, determined as before, is given by $-\Delta \lambda=A \alpha_{1}^{2}+B \alpha_{2}^{2}+C \alpha_{3}^{2}+2 F \alpha_{2} \alpha_{3}+2 G \alpha_{3} \alpha_{1}+2 H \alpha_{1} \alpha_{2}=E$, say ; the usual notation being employed for the discriminant of $S$ and its minors. Assuming, as we may without loss,

and writing

$$
q_{3}=0, \Delta \mu p_{3}=1 \text {, }
$$

$$
2 E_{r}=\frac{\partial E}{\partial a_{r}},(r=1,2,3),
$$

we find without dificulty

$$
\left.\begin{array}{ll}
E\left(l_{13}-\cos \theta\right) & =\alpha_{1} E_{1}(1-\cos \theta)+\left(g \alpha_{2}-h \alpha_{3}\right) \sqrt{ } E \cdot \sin \theta \\
E l_{12} & =\alpha_{2} E_{1}(1-\cos \theta)+\left(f \alpha_{2}-b \alpha_{3}\right) \sqrt{ } E \cdot \sin \theta \\
E l_{13} & =\alpha_{3} E_{1}(1-\cos \theta)+\left(c \alpha_{2}-f \alpha_{3}\right) \sqrt{ } E \cdot \sin \theta
\end{array}\right\}
$$

together with the six others derived from these by simultaneous cyclic interchange of the suffixes $1,2,3$, and the two sets of symbols $a, b, c ; f, g, h$. It is evident that, for a real substitution, $E$ is necessarily positive.

The process here employed is analogous to that which arises in finding in homogeneous plane point-coordinates the (imaginary) tangents to the conic $S$ at its points of intersection with the line $\xi_{3}$; or in finding in three-dimensional cartesian coordinates the tangent planes to the quadric cone $S$ which touch along the generators in which the cone is cut by the plane $\xi_{3}$. The condition that $E$ is positive ensures that the points of intersection in the first case, and the lines of intersection in the second, shall be imaginary, and therefore also the tangent lines and planes:

The substitution might be interpreted geometrically as follows. Let

$$
S=\text { const., } \xi_{3}=\text { const. }
$$

denote respectively a conicoid and a plane in trirectangular coordinates; let $Q$ be any point on their curve of intersection, and $O N$ the normal to the plane from the centre of the surface. Let $Q$ move along the curve of intersection until the plane $O N Q$ has rotated through an angle $\theta$ about $O N$; then the direction-cosines of the new position of $O Q$ are obtained from those of the old by the substitution whose coefficients $l_{r}$ are given in (26).

The generality of the result would not be impaired by assuming $E=1$; and we would then have

$$
\alpha_{1} E_{1}+\alpha_{2} E_{2}+\alpha_{3} E_{3}=E=1 \text {. }
$$


§8. $n=4$.

Generalising the case of four variables already treated in $\$ 5$, let us take as the invariants of a real substitution in four variables, with equimodular multipliers, the following :

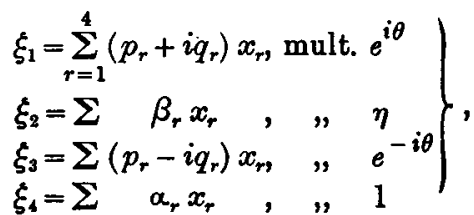

where the $a, \beta, p, q$ are real, and $\eta$ denotes $\mp 1$ according as morle (A) or (B) is under consideration.

The coordinates of the poles, and the formulae for the coetticients of the substitution, can be obtained exactly as in the case of $n=3$. Taking as coordinates the actual first minors of the determinant of the coefficients of $(27)$, and writing

we obtain for the poles :

$$
\left(p_{2} \beta_{3} \alpha_{4}\right) \equiv\left|\begin{array}{lll}
p_{2}, & p_{3}, & p_{4} \\
\beta_{2}, & \beta_{3}, & \beta_{4} \\
\alpha_{2}, & \alpha_{3}, & \alpha_{4}
\end{array}\right| \text {, etc. }
$$

$P_{1}\left\{-\left(p_{2} \beta_{3} \alpha_{4}\right)+i\left(q_{2} \beta_{3} \alpha_{4}\right),\left(p_{1} \beta_{3} \alpha_{4}\right)-i\left(q_{1} \beta_{3} \alpha_{4}\right),-\left(p_{1} \beta_{2} \alpha_{4}\right)+i\left(q_{1} \beta_{2} \alpha_{4}\right),\left(p_{1} \beta_{2} \alpha_{3}\right)-i\left(q_{1} \beta_{2} \alpha_{3}\right)\right\}$ $P_{2}\left\{2 i\left(p_{2} q_{3} \alpha_{4}\right) \quad, \quad-2 i\left(p_{1} q_{3} \alpha_{4}\right), \quad 2 i\left(p_{1} q_{2} \alpha_{4}\right) \quad, \quad-2 i\left(p_{1} q_{2} \alpha_{3}\right)\right.$

$P_{3}\left\{\left(p_{2} \beta_{3} \alpha_{4}\right)+i\left(q_{2} \beta_{3} \alpha_{4}\right)\right.$,

$P_{4} 1-2 i\left(p_{2} q_{3} \beta_{4}\right)$

$2 i\left(p_{1} q_{3} \beta_{4}\right)$,

$2 i\left(p_{1} q_{2} \beta_{4}\right)$

$2 i\left(p_{1} q_{2} \beta_{3}\right)$

those of $P_{3}$ being obtained from $P_{1}$ by changing the signs of the real parts. Write

$$
D_{0}=\left|\begin{array}{cccc}
p_{1}, & p_{2}, & p_{3}, & p_{\phi} \\
\beta_{1}, & \beta_{2}, & \beta_{3}, & \beta_{4} \\
q_{1}, & q_{2}, & q_{3}, & q_{4} \\
\alpha_{1}, & \alpha_{2}, & \alpha_{3}, & \alpha_{4}
\end{array}\right| ;
$$

then the determinant of the coefficients of $(27)$ is $2 i D_{0}$, and that of the coordinates just set down is

$$
\left(-2 i D_{0}\right)^{3}=8 i D_{0}{ }^{3}=D, \text { say. }
$$

If we denote by $X_{r \varepsilon}$ as usual the appropriate first minor of $D$, then it is clearly equal to $-4 D_{0}^{2}$ times the corresponding coefficient in (27). Now applying formula (5), $D l_{r n}=\left(x_{r 1} X_{a 1}+x_{r 3} X_{s 3}\right) \cos \theta+\left(x_{r 1} X_{t 1}-x_{r 3} X_{t 3}\right) i \sin \theta+\eta x_{r 2} X_{s 2}+x_{r 4} X_{4}$. 
Denoting the coefficients of (27) for convenience by

we can put

$$
c_{r s}(r, s=1,2,3,4) \text {, }
$$

$$
\left.\begin{array}{l}
2 D_{0} l_{r s}=A_{r s} \cos \theta+B_{r s} \sin \theta+C_{r s},(\text { mode A) } \\
2 D_{0} l_{r s}=A_{r t} \cos \theta+B_{r s} \sin \theta+D_{r s},(\text { mode B) }
\end{array}\right\}
$$

where

$$
\left.\begin{array}{ll}
A_{r s}=i\left(x_{r 1} c_{t 1}+x_{r 3} c_{r 3}\right), & C_{r s}=-i\left(x_{r 3} c_{r 2}-x_{r 4} c_{s 4}\right) \\
B_{r t}=-\left(x_{r 1} c_{t 1}-x_{r 3} c_{t 3}\right), & D_{r s}=i\left(x_{r 2} c_{r 3}+x_{r 4} c_{t 4}\right)
\end{array}\right\} \ldots
$$

It would be tedious to express the 64 coefficients at length in terms of the $\alpha, \beta, p, q$; but we shall find them for the special case which is analogous to rotation about any axis in three dimensions.

Mode $(A)$.-An important divergence from the case of $n=3$ arises from the existence, in the present case, of two real linear invariants $\xi_{2}=\Sigma \beta x, \xi_{4}=\Sigma \alpha x$. For there are now three independent real quadratic invariants with multiplier +1 , namely,

$$
\xi_{2}^{2}, \xi_{4}^{2}, \xi_{1} \xi_{3} \text {. }
$$

If therefore on the analogy of last article we assume $\Sigma x^{2}$ as an invariant in addition to $\xi_{3}, \xi_{4}$, the relation from which the $p_{n}, q_{r}$ of $\xi_{1}, \xi_{3}$ are to be found is of the form

$$
(\Sigma \alpha x)^{2}+\nu(\Sigma \beta x)^{2}+\lambda \Sigma x^{2}=\mu\left\{(\Sigma p x)^{2}+(\Sigma q x)^{2}\right\} \ldots \ldots
$$

Now Sylvester's Law of Inertia states that when a quadratic form is expressed as a sum of squares, the number of positive squares and the number of negative squares are fixed; hence in (30) $\lambda, \mu$ must be negative and $\nu$ positive.

We may (by reducing the $\beta$, if necessary, in a constant ratio) assume $v=+1$. The condition that the left-hand member shall break, like the right-hand, into linear factors, is* that not only its discriminant, but also every first minor thereof, shall vanish. The discriminant is a biquadratic in $\lambda$, of which the absolute term and the coefficient of the first power of $\lambda$ are easily seen to be zero. Rejecting the double zero root we are left with a quadratic. But $\dagger$ this quadratic must have equal roots ; hence there is only one suitable value of $\lambda$.

- Salmon-Rogers, Analytical Geometry of Three Dimensions (1912), $\$ 79$.

† See, e.g., Routh, Advanced Rigid Dynamic8, § 269. 
Writing

$$
\alpha_{r} \alpha_{s}+\beta_{r} \beta_{s}=\gamma_{r s},
$$

one of the first minors is

$$
\left|\begin{array}{lll}
\gamma_{21}, & \gamma_{22}+\lambda, & \gamma_{23} \\
\gamma_{31}, & \gamma_{32}, & \gamma_{33}+\lambda \\
\gamma_{31}, & \gamma_{42}, & \gamma_{43}
\end{array}\right| ;
$$

this, equated to zero, yields the zero root and another, the one we are in search of, viz.,

$$
\begin{aligned}
\lambda & =-\gamma_{22}-\gamma_{33}+\frac{\gamma_{31} \gamma_{43}+\gamma_{21} \gamma_{42}}{\gamma_{41}} \\
& =-\left(\gamma_{11}+\gamma_{92}+\gamma_{33}+\gamma_{44}\right)+\frac{\alpha_{1} \alpha_{4} \Sigma \alpha^{2}+\left(\alpha_{1} \beta_{4}+\alpha_{4} \beta_{1}\right) \Sigma \alpha \beta+\beta_{1} \beta_{4} \Sigma \beta^{2}}{\alpha_{1} \alpha_{4}+\beta_{1} \beta_{4}} .
\end{aligned}
$$

Since a similar relation must hold for every other pair of suffixes as well as 1, 4, we must have

$$
\Sigma \alpha^{2}=\Sigma \beta^{2}, \quad \Sigma \alpha \beta=0,
$$

whence $-\lambda=\Sigma \alpha^{2}=\Sigma \beta^{2}$. On equating coefficients in (30) we now have 10 conditions; which are satisfied by the following values:

$$
\begin{aligned}
& p_{4}=-\left(\alpha_{1}{ }^{2}+\alpha_{2}{ }^{2}+\alpha_{3}{ }^{2}-\beta_{4}{ }^{2}\right)=\mu^{-1}, \\
& p_{1}=\alpha_{1} \alpha_{4}+\beta_{1} \beta_{4}, \\
& p_{2}=\alpha_{2} \alpha_{4}+\beta_{2} \beta_{4}, \\
& p_{3}=\alpha_{3} \alpha_{4}+\beta_{8} \beta_{4} \\
& q_{1}=\alpha_{2} \beta_{3}-\alpha_{3} \beta_{3} \\
& q_{2}=\alpha_{3} \beta_{1}-\alpha_{1} \beta_{3}, \\
& q_{3}=\alpha_{1} \beta_{2}-\alpha_{2} \beta_{1}, \\
& q_{4}=0 .
\end{aligned}
$$

It will also be found that there is no loss of generality in taking these values as the general solution.

For the further discussion of this case we may assume

$$
-\lambda \equiv \Sigma \alpha^{2} \equiv \Sigma \beta^{2}=1 \text {, whence } p_{4}=\alpha_{4}{ }^{2}+\beta_{4}^{2}-1 .
$$

Referring back to the coordinates of the poles $P_{r}$ we can prove

$$
\left(p_{2} \beta_{3} a_{4}\right)=q_{1},\left(q_{2} \beta_{3} \alpha_{4}\right)=-p_{1} \text {, etc., }
$$


hence the coordinates take the simple forms

$$
\begin{aligned}
& P_{1}: \frac{x_{11}}{-i\left(p_{1}-i q_{1}\right)}=\frac{x_{21}}{-i\left(p_{2}-i q_{2}\right)}=\frac{x_{31}}{-i\left(p_{3}-i q_{3}\right)}=\frac{x_{41}}{-i\left(p_{4}-i q_{4}\right)}, \\
& P_{2}: \frac{x_{12}}{2 i p_{4} \beta_{1}}=\frac{x_{22}}{2 i p_{4} \beta_{2}}=\frac{x_{32}}{2 i p_{4} \beta_{3}}=\frac{x_{42}}{2 i p_{4} \beta}, \\
& P_{8}: \frac{x_{13}}{-i\left(p_{1}+i q_{1}\right)}=\frac{x_{23}}{-i\left(p_{2}+i q_{2}\right)}=\frac{x_{33}}{-i\left(p_{3}+i q_{3}\right)}=\frac{x_{43}}{-i\left(p_{4}+i q_{4}\right)}, \\
& P_{4}: \frac{x_{14}}{2 i p_{4} \alpha_{1}}=\frac{x_{24}}{2 i p_{4} \alpha_{2}}=\frac{x_{34}}{2 i p_{4} \alpha_{3}}=\frac{x_{44}}{2 i p_{4} \alpha_{4}},
\end{aligned}
$$

the common factors being retained for convenience. Also

$$
D_{0}=-p_{4} \text {; }
$$

hence finally the coefficients of the substitution in mode (A) for which $\Sigma \alpha x, \Sigma \beta x, \Sigma x^{2}$ are invariant are given by

$$
l_{r s}=a_{r s} \cos \theta+b_{r s} \sin \theta+c_{r s},
$$

where

$$
\begin{aligned}
-a_{r s} & =a_{r} \alpha_{s}+\beta_{r} \beta_{s}, r \neq s, \\
-a_{r r} & =\alpha_{r}^{2}+\beta_{r}^{2}-1, \\
c_{r s} & =a_{r} \alpha_{s}-\beta_{r} \beta_{s},
\end{aligned}
$$

and the $b_{r a}$ are given in the following scheme, the arrangement as to the suffixes being the same as in the table of coordinates of poles just above :

$$
\begin{array}{ccccc}
0 & -\left(\alpha_{3} \beta_{4}\right), & \left(\alpha_{2} \beta_{4}\right), & -\left(\alpha_{2} \beta_{3}\right), \\
b_{r 8}: & \left.\alpha_{3} \beta_{4}\right), & 0 & -\left(\alpha_{1} \beta_{4}\right), & -\left(\alpha_{3} \beta_{1}\right), \\
-\left(\alpha_{2} \beta_{4}\right), & \left(\alpha_{1} \beta_{4}\right), & 0 & -\left(\alpha_{1} \beta_{2}\right), \\
\left(\alpha_{2} \beta_{3}\right), & \left(\alpha_{3} \beta_{1}\right), & \left(\alpha_{1} \beta_{2}\right), & 0,
\end{array}
$$

where $\left(\alpha_{r} \beta_{s}\right) \equiv \alpha_{r} \beta_{1}-\alpha_{\alpha} \beta_{r}$. It can at once be verified that these values agree with those given in $\$ 5$ (A) for the special case

$$
\alpha_{1}=\alpha_{2}=\alpha_{3}=\alpha_{4}=-\beta_{1}=\beta_{2}=-\beta_{3}=\beta_{4}=\frac{1}{2} \text {. }
$$

We have thus completed the determination of the substitution in four variables in mode (A) analogous to that which represents a rotution about a given axis in three dimensions.

Mode $(B)$. - If for the case of mode (B) we were to make the same assumption as in (30), we would find that the alteration noeded in going from (A) to (B), equivalent to the change from $C_{r a}$ to $D_{r}$ in (29), amounts simply to changing the minus signs in 
the formula for the $c_{r, s}$ above into plus. It will have been noted that the formulae for the $h$ in modes (A), (B) (\$5) differ only in the constant terms.

But it appears that the assumption (30) is not sufficiently general for mode $(B)$, since a term of the form

$$
\eta \Sigma(\alpha x) \Sigma(\beta x), \quad(\eta \text { const. })
$$

might also have place on the left. To determine whether this would introduce any more generality into the result, it will be sufficient to replace the $\alpha_{r}, \beta_{r}$ in (23) by

$$
\alpha_{r}^{\prime}=l \alpha_{r}+l^{\prime} \beta_{r} \quad \beta_{r}^{\prime}=m \alpha_{r}+m^{\prime} \beta_{r}, \quad(r=1,2,3,4),
$$

where we may obviously assume

$$
-\lambda=\Sigma \alpha_{r}^{\prime 2}=\Sigma \beta_{r}^{\prime 2}, \Sigma \alpha_{r}^{\prime} \beta_{r}^{\prime}=0 .
$$

These relations yield

whence (say)

$$
l^{2}+l^{\prime 2}=m^{2}+m^{\prime 2}=1, l l^{\prime}+m m^{\prime}=0,
$$

$$
\begin{gathered}
l=\cos \zeta, l^{\prime}=\sin \zeta, m=-\sin \zeta, m^{\prime}=\cos \zeta, \\
l^{2}+m^{2}=l^{\prime 2}+m^{\prime 2}=1, l m^{\prime}-l^{\prime} m=1 .
\end{gathered}
$$

Denoting the new coefficients of the imaginary invariants by accented letters $p_{r}^{\prime}, q_{r}^{\prime}$, we have

$$
\begin{aligned}
p_{4}^{\prime} & =\left(l \alpha_{4}+l^{\prime} \beta_{4}\right)^{2}+\left(m \alpha_{4}+m^{\prime} \beta_{4}\right)^{2}+\lambda \\
& =\alpha_{4}^{2}+\beta_{4}^{2}+\lambda \\
& =p_{4},
\end{aligned}
$$

so that no generality is lost in the case of mode (B) by omission of the product term from the left of (30). A similar proof will hold for the more general case treated below.

$\$ 9$. Suppose that in place of $\Sigma x^{2}$ we are given as quadratic invariant the general quadratic form

$$
\left.S \equiv(a, b, c, d, f, g, h, l, m, n\rangle x_{1}, x_{i}, x_{i}, x_{\downarrow}\right)^{2},
$$

It is known (Bronwich, Quadratic Forms, $\$ 8$ ) that this can be written

$$
S=\frac{\zeta_{1}^{2}}{a}+\frac{\zeta_{2}^{2}}{a C}+\frac{\zeta_{3}^{2}}{C \Delta}+\frac{\zeta_{4}^{2}}{\Delta D}
$$

where the $\zeta$ are linear functions of the $x, D$ is the discriminant of the given form, and $\Delta, C$ are obtained in succession from $D$ by removals of the last row and column. 
In the case of an odd number of variables, if the discriminant of the form were negative, we could consider instead the same form affected with the minus sign, of which the discriminant is positive. But when, as here, the number of variables is even, the discriminants of $S,-S$ are identical, and we must consider two cases, according as $D$ is positive or negative.

\section{I. $D$ positive.}

Here, by Sylvester's law of inertia, $S$ must either be a sum of four positive squares, or of two positive and two negative, or of four negative, according to the signs of $a, C, \Delta$. We may therefore make an assumption of the form

$$
(\Sigma \alpha x)^{2}+(\Sigma \beta x)^{2}+\lambda S=\mu\left\{(\Sigma p x)^{2}+(\Sigma q x)^{2}\right\} .
$$

\section{II. $D$ negative.}

Here $S$ is either a sum of three positive and one negative squares, or of one positive and three negative, and our assumption must be of the form

$$
(\Sigma x x)^{2}-(\Sigma \beta x)^{2}+\lambda S=\mu\left\{(\Sigma p x)^{2}+(\Sigma q x)^{2}\right\} .
$$

Results for the second case may, then, be deduced from those for the first by affecting each $\beta$ that occurs with the coefficient $i$.

We can follow the method of the less general case $(\$ 8)$ up to a certain point. Let us put

$$
\phi_{1} \equiv\left|\begin{array}{ccccc}
a, & h, & g, & l, & \alpha_{1} \\
h, & b, & f, & m, & \alpha_{2} \\
g, & f, & c, & n, & \alpha_{3} \\
l, & m, & n, & d, & \alpha \\
\alpha_{1}, & \alpha_{2}, & \alpha_{3}, & \alpha_{4}, & 0
\end{array}\right| ;
$$

let $\phi_{2}$ denote the corresponding expression when the $\alpha$ in the last row and column are replaced by the corresponding $\beta$, and $\phi_{12}$ the expression when the $\alpha$ of the last row, but not of the last column, are replaced by the $\beta$.

The discriminant of the left-hand member of (34) is, as before, a biquadratic in $\lambda$ with two roots zero, and the residuary quadratic is where

$$
D \lambda^{2}+\left(\phi_{1}+\phi_{2}\right) \lambda+\psi=0 \text {, }
$$

Since this quadratic must, as before, have equal roots, hence

and

$$
\begin{aligned}
\phi_{1} & =\phi_{2}, \phi_{12}=0, \ldots \\
& -D \lambda=\phi_{1}=\phi_{2} .
\end{aligned}
$$


Using $\phi$ to denote the common value of $\phi_{1}, \phi_{2}$ we have for the $p, q$ ten relations typified by the following:

$$
\left.\begin{array}{l}
D\left(\alpha_{1}{ }^{2}+\beta_{1}{ }^{2}\right)-\phi a=\mu D\left(p_{1}{ }^{2}+q_{1}{ }^{2}\right) \\
D\left(\alpha_{2} \alpha_{3}+\beta_{2} \beta_{3}\right)-\phi f=\mu D\left(p_{2} p_{3}+q_{2} q_{3}\right) \\
D\left(\alpha_{1} \alpha_{4}+\beta_{1} \beta_{4}\right)-\phi l=\mu D\left(p_{1} p_{4}+q_{1} q_{4}\right)
\end{array}\right\}
$$

Assuming $q_{4}=0, \mu D p_{4}=1$, we thence obtain $p_{1}, p_{2}, p_{3}, p_{4}$ as rational quadratic functions of the $\alpha_{1} \beta$; and for $q_{1}, q_{2}, q_{3}$ equations such as

$$
\left.\begin{array}{rl}
p_{1}{ }^{2}+q_{1}{ }^{2} & =\left\{D\left(\alpha_{1}{ }^{2}+\beta_{1}{ }^{2}\right)-\phi a\right\}\left\{D\left(\alpha_{4}{ }^{2}+\beta_{4}{ }^{2}\right)-\phi d\right\} \\
p_{2} p_{3}+q_{2} q_{3} & =\left\{D\left(\alpha_{2} \alpha_{3}+\beta_{2} \beta_{3}\right)-\phi f\right\}\left\{D\left(\alpha_{4}{ }^{2}+\beta_{4}{ }^{2}\right)-\phi d\right\}
\end{array}\right\}
$$

It is clear from the relation

$$
\phi^{2}=\phi_{1} \phi_{2}-\phi_{12}{ }^{2}
$$

and the forms of the expressions on the right hand of this identity, that $\phi^{2}$ contains the factor $D$. It therefore follows from (37) that $q_{1}, q_{2}, q_{3}$ are each of the form

$$
\left.D^{\frac{1}{3}} \times \text { (rational quadratic function of the } a, \beta\right) \text {. }
$$

The labour of obtaining the actual expressions can be avoided by turning to the geometrical interpretation of the analytical process; and this will also throw new light on the meaning of the coefficieuts $p, q$.

In the phraseology of the theory of the six coordinates of a line (see Salmon-Rogers, Analytical Geometry of Three Dimensions, $\$ 53 f f$.$) , let s_{r}, \sigma_{r}(r=1,2, \ldots 6)$ denote respectively the raycoordinates and axial-coordinates of the line of intersection of the planes

$$
\Sigma \alpha x=0, \Sigma \beta x=0 ;
$$

let $s_{r}{ }^{\prime}, \sigma_{r}^{\prime}$ denote the corresponding coordinates of the line of intersection of the planes

$$
\Sigma p x=0, \Sigma q x=0
$$

and let these lines be called respectively (1), (2). Then with the abbreviations

we have

$$
\left(\alpha_{r} \beta_{s}\right) \equiv\left(\alpha_{r} \beta_{s}-\alpha_{s} \beta_{r}\right), \text { etc., }
$$

$$
\left.\begin{array}{l}
\left(\alpha_{2} \beta_{3}\right)=\sigma_{1}=s_{4},\left(\alpha_{3} \beta_{1}\right)=\sigma_{2}=s_{5}, \quad\left(\alpha_{1} \beta_{2}\right)=\sigma_{3}=s_{6} \\
\left(\alpha_{1} \beta_{4}\right)=\sigma_{4}=s_{1},\left(\alpha_{2} \beta_{4}\right)=\sigma_{5}=s_{2},\left(\alpha_{3} \beta_{4}\right)=\sigma_{6}=s_{3} \\
\left(p_{2} q_{3}\right)=\sigma_{1}^{\prime}=s_{4}^{\prime}, \text { etc. } \\
\left(p_{1} q_{4}\right)=\sigma_{4}^{\prime}=s_{1}^{\prime}, \text { etc. }
\end{array}\right\}
$$

Now it is clear from (34) that the planes $\Sigma \alpha x, \Sigma \beta x, \Sigma p x, \Sigma q x$ form a tetrahedron self-conjugate with respect to the conicoid $S$, 
and therefore that (2) is the polar line of line (1). But the coordinates of the polar of a given line with respect to a conicoid can be obtained as follows.

Let $a_{r s}(r, s=1,2, \ldots 6)$ denote (see Salmon-Rogers, $\$ 80 c$ ) the second minors of the discriminant of $S$, and let

$$
\Psi \equiv\left(a_{11}, \ldots a_{56} \chi s_{1}, s_{2}, \ldots s_{6}\right)^{2} ;
$$

then unaccented letters denoting the coordinates of the given line, and accented letters those of the required line,

$$
2 \sigma_{r}{ }^{\prime}=\frac{\partial \Psi}{\partial s_{r}},(r=1,2, \ldots 6) .
$$

With the single assumption $q_{4}=0$, we have

$$
-\sigma_{4}^{\prime}=p_{4} q_{1}, \quad-\sigma_{5}^{\prime}=p_{4} q_{9}, \quad-\sigma_{6}^{\prime}=p_{4} q_{3} ;
$$

and if in (36) we further assume $\mu D=1$, we at once obtain the values of

we thus have the ratios

$$
p_{4} p_{1}, p_{4} p_{2}, p_{4} p_{3,}, p_{4}^{2} ;
$$

$$
p_{1}: p_{2}: p_{3}: p_{4}: q_{1}: q_{2}: q_{3}
$$

as required. We may write the results, subject to the insertion of an arbitrary constant factor, as follows :

$$
\begin{aligned}
& p_{1}=D\left(\alpha_{1} \alpha_{4}+\beta_{1} \beta_{4}\right)-l \phi \text {, } \\
& p_{2}=D\left(\alpha_{2} \alpha_{4}+\beta_{2} \beta_{4}\right)-m \phi \text {. } \\
& p_{3}=D\left(\alpha_{3} \alpha_{4}+\beta_{3} \beta_{4}\right)-n \phi \text {, } \\
& p_{4}=D\left(\alpha_{4}^{2}+\beta_{4}^{2}\right)-d \phi \text {, } \\
& q_{1}=-\left|\begin{array}{llll}
a, & h, & g, & l \\
\alpha_{1}, & \alpha_{2}, & \alpha_{3}, & \alpha_{4} \\
\beta_{1} & \beta_{2}, & \beta_{3}, & \beta_{4} \\
l, & m, & n, & d
\end{array}\right|, \quad q_{2}=+\left|\begin{array}{llll}
\alpha_{1}, & \alpha_{2}, & \alpha_{3}, & \alpha_{4} \\
h, & b, & f, & m \\
\beta_{1}, & \beta_{2}, & \beta_{3}, & \beta_{4} \\
l, & m, & n, & d
\end{array}\right|, \\
& q_{3}=-\left|\begin{array}{llll}
\alpha_{1}, & \alpha_{2}, & \alpha_{3}, & \alpha_{4} \\
\beta_{1}, & \beta_{2}, & \beta_{3}, & \beta_{4} \\
g, & f, & c, & n \\
l, & m, & n, & d
\end{array}\right|
\end{aligned}
$$

\$10. Finally, we can now indicate the answer to another question, viz.,

Under what conditions will two real quadratic invariants serve to define a real equimodular substitution in four variables? 
If $S, S^{\prime \prime}$ are the given invariants, it is necessary in order to obtain the requisite four linear invariants that $S+\lambda S^{\prime \prime}$ shall break up into two planes (real or imaginary) for two distinct values of $\lambda$. For each of these values therefore the discriminant of $S+\lambda S^{\prime \prime}$ and all its first minors must vanish.

Now in the classification of quadratic forms in four variables (see Bromwich, loc. cit., $\S 18$ ), the only case satisfying these conditions is that numbered 9 on Lindemann's list, with the symbol [(11) (11)]; namely that in which $S, S^{\prime \prime}$ have four generators in common, forming a skew quadrilateral, at the vertices of which the conicoids touch.* We can then write

$$
\begin{aligned}
S & =a_{1} \zeta_{1}^{2}+a_{2} \zeta_{2}^{2}+a_{3} \zeta_{3}^{2}+a_{4} \zeta_{4}^{2}, \\
S^{\prime \prime} & =c_{1}\left(a_{1} \zeta_{1}^{2}+a_{2} \zeta_{2}^{2}\right)+c_{3}\left(a_{3} \zeta_{3}^{2}+a_{4} \zeta_{4}^{2}\right),
\end{aligned}
$$

where the $\zeta$ are linear functions of the coordinates; the one pair of linear invariants will then be linear functions of $\zeta_{1}, \zeta_{2}$; and the other pair, of $\zeta_{3}, \zeta_{4}$, Of these invariants there will be four, two, or none real according to the signs of the coefficients $a_{n}$ which are functions of the coefficients of the forms.

Thus, assuming the forms to satisfy the above conditions, and reducing them to sums of squares by the method given by Bromwich (\$17), we immediately obtain the linear invariants, from which the coefficients of the substitution can be derived by the method of $\$ 8$ above. The variety of possible cases would probably render unprofitable the search for a general formula like that of the last article. The only novelty is the case in which all four linear invariants, and therefore also the multipliers, are imaginary; they consist, of course, of conjugate pairs, and the treatment offers no difficulties.

* See also Bell, Coordinate Geometry of Three Dimensions, \$\$ 166-8; Salmon-Rogers, § 202. 